\title{
Relation between chronic endometritis and recurrent miscarriage
}

\author{
Mohamed M. Farghali, ${ }^{1,2}$, Ibrahim A. Abdelazim ${ }^{1,3}$, Tamer E. El-Ghazaly ${ }^{1}$ \\ 1Department of Obstetrics and Gynecology, Ain Shams University, Cairo, Egypt \\ 2Department of Obstetrics and Gynecology, Sabah Maternity Hospital, Kuwait \\ ${ }^{3}$ Department of Obstetrics and Gynecology, Ahmadi Hospital, Kuwait Oil Company (KOC), Kuwait
}

\begin{abstract}
Introduction: Growing interest has focused on the relation between chronic endometritis (CE) or asymptomatic inflammation of the endometrium and recurrent miscarriage (RM).

The aim of the study was to assess the relation between CE and RM and the accuracy of hysteroscopy in diagnosing $\mathrm{CE}$.

Material and methods: One hundred and ten (110) women with unexplained RM were included in this study. Participants were subjected to diagnostic hysteroscopy for uterine cavity, and endometrium evaluation. The diagnosis of CE during hysteroscopic examination was based on CE-related hysteroscopic signs (micropolyps, stromal edema, and/or hyperemia). At the end of hysteroscopy, an endometrial biopsy was taken from participants for culture, and immunohistochemical (IHC) staining. Collected data were analyzed to assess the relation between CE and RM and the accuracy of hysteroscopy in diagnosing CE.

Results: The prevalence of CE in women with RM was $31.8 \%$ using CE-related hysteroscopic signs, while it was $38.2 \%$ using IHC staining and endometrial cultures ( $p=0.4$ ). CE-related hysteroscopic signs had $64.1 \%$ sensitivity, $85.9 \%$ specificity, $71.4 \%$ positive predictive value (PPV), $81.3 \%$ negative predictive value (NPV), and $78.2 \%$ overall accuracy in diagnosing CE. Most cases of CE (> 81\%) were caused by Mycoplasma and common pathogens.

Conclusions: The prevalence of CE in women with RM was 31.8\% using CE-related hysteroscopic signs, while it was $38.2 \%$ using IHC staining and endometrial cultures. CE-related hysteroscopic signs had $64.1 \%$ sensitivity, $85.9 \%$ specificity, $71.4 \%$ PPV, $81.3 \%$ NPV, and $78.2 \%$ overall accuracy in diagnosing CE. Most cases of CE (> 81\%) were caused by Mycoplasma and common pathogens.
\end{abstract}

Key words: endometritis, recurrent, miscarriage.

\section{Introduction}

Recurrent miscarriage (RM) or recurrent pregnancy loss (RPL) is defined as $\geq 3$ pregnancy losses before the age of fetal viability [1, 2]. Pregnancy loss is a negative life event, and RM may intensify the grief experienced [1].

Genetic abnormalities, endocrine disorders, anti-phospholipid syndrome (APS), and uterine abnormalities were detected in $50 \%$ of recurrent miscarriages (RMs), while the remaining $50 \%$ of RMs were considered unexplained RMs [1-3].

Growing interest has been focused on the relation between chronic endometritis (CE) or asymptomatic inflammation of endometrium and RM [4].

The reported prevalence of CE in women with RM differs between studies. Kimura et al. reported a 9.3-67.6\% prevalence of CE in RMs [5], while Bouet et al. reported a $27 \%$ prevalence of CE in RMs [6].

The inflammatory cells are normally present in endometrium; therefore, histologic examination of the endometrium may miss the diagnosis of CE $[1,4]$.
Identification of plasma cells in the endometrial stroma associated with pathogenic organisms is the gold standard for diagnosing CE $[3,6-8]$.

Previous studies concluded that the hysteroscopic diagnosis of CE should be based on identification of micro-polyps (<2 mm), stromal edema, hyperemia (focal or diffuse), or hemorrhagic spots during uterine cavity and endometrium evaluation [9-12].

Therefore, this study was designed to assess the relation between $\mathrm{CE}$ and $\mathrm{RM}$ and the accuracy of $\mathrm{CE}$-related hysteroscopic signs (micropolyps, stromal edema, and hyperemia) in diagnosing $\mathrm{CE}$.

\section{Material and methods}

The hospital records were reviewed over 5 years from January 2012 to January 2017 to identify women with unexplained RM or RPL after approval of the Obstetrics and Gynecology department, and trial registration [13]. 
One hundred and ten (110) women aged 20-40 years, with unexplained RM before the age of fetal viability following spontaneous or ART conception, were included in this comparative double-blinded study after obtaining informed consent in accordance with the Declaration of Helsinki.

RM is defined as $\geq 3$ pregnancy losses [1, 2]. A pregnancy loss (miscarriage) is defined as spontaneous demise of pregnancy before the age of fetal viability (24 weeks' gestation) [1, 2].

RM includes early pregnancy loss or biochemical pregnancy loss ( $>6$ weeks) after diagnosis of pregnancy by $\beta$-hCG (serum or urine), and an ultrasound scan was only done after complete expulsion of the embryo and trophoblasts. It also includes clinical miscarriage or clinical pregnancy loss after diagnosis of pregnancy by an ultrasound scan [1]. Recurrent miscarriage does not include ectopic, molar pregnancies, and/or implantation failure [1].

Two types of unexplained RM were defined by Saravelos et al.; type I occurs by chance in women who have no underlying pathology (has a good prognosis), and type II occurs due to an underlying pathology that is not currently identified by routine investigations (has a poor prognosis) [14].

Exclusion criteria include: 1) obese (body mass index $\geq 30 \mathrm{~kg} / \mathrm{m}^{2}$ ), and/or alcoholic women, 2) women with family history of RM, history of uterine abnormalities, previous uterine surgery approaching the cavity (i.e., myomectomy), atypical endometrial hyperplasia or complex hyperplasia, and/or endocrine or metabolic disorders, 3) women with previous history of endometriosis and/or endometritis (diagnosed clinical and/or by ultrasound), autoimmune diseases (i.e., APS), and/or thrombophilia, 4) women on anticoagulant therapy, antibiotics, oral contraceptive within the last 3 months, 5) women who refused to participate, and/or give consent.

Studied women were subjected to diagnostic hysteroscopy in the follicular phase of menstrual cycle (5-7 days post-menstrually) under general anesthesia using Bettocchi $5 \mathrm{~mm}$, 30-degree hysteroscopy (Karl Storz, Germany), and normal saline as distension medium [15].

During hysteroscopies, the uterine cavity and endometrium were thoroughly evaluated (from the cervix to the uterine fundus and both cornua) to identify CE-related hysteroscopic signs. The diagnosis of CE was based on the presence of $\geq 1$ CE-related hysteroscopic signs, which include micro-polyps $(<2 \mathrm{~mm})$ that fluctuate in the cavity (Fig. 1), stromal edema, and hyperemia (focal or diffuse) (Fig. 2) [9-12].

The reordered hysteroscopic findings were evaluated by senior consultant ( $\geq 12$ years' experience in hysteroscopic surgery after the MD degree), who was blinded to participants' clinical data (to avoid potential bias).

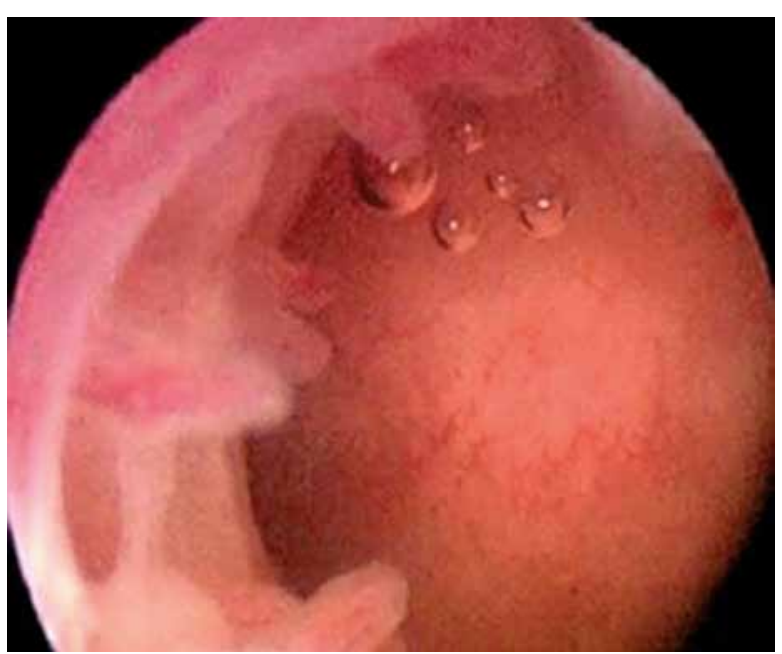

Fig. 1. Chronic endometritis-related hysteroscopic sign (micropolyps)

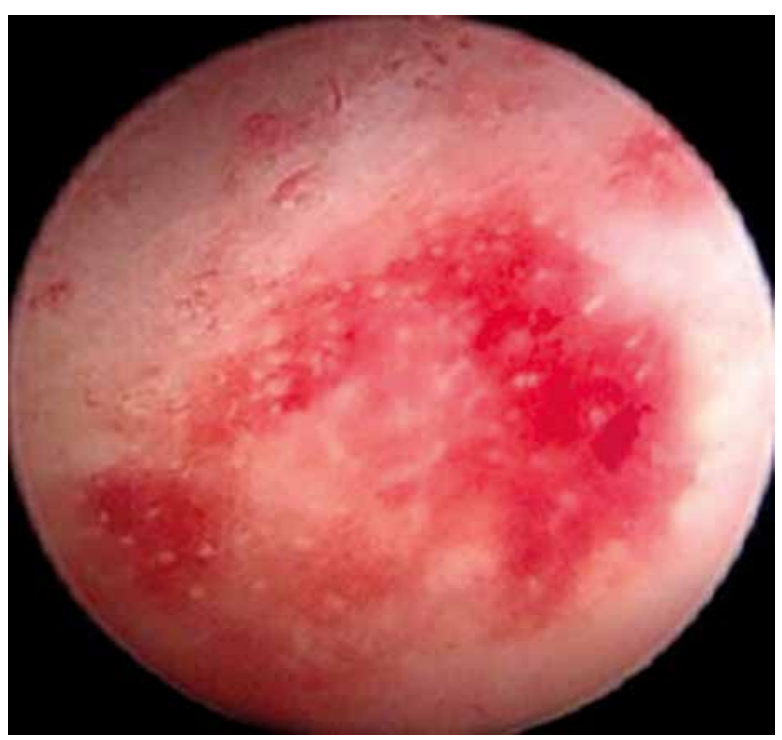

Fig. 2. Chronic endometritis-related hysteroscopic sign (focal hyperemia)

At the end of hysteroscopic procedures an endometrial biopsy was taken from each participant using a 5-mm flexible Karman cannula (MedGyn, USA) connected to its aspiration kit.

Each endometrial biopsy divided into 2 samples: one placed in saline for culture, and the other placed in formalin for immunohistochemical (IHC) staining [5].

The endometrial biopsies taken for IHC staining were mounted on 3-aminopropyl-trethoxysilane coated slides, stained with monoclonal anti-human CD138 against plasma cells, and examined by an expert who was blinded to participants' clinical data (double-blinded) to detect the CD138-positive plasma cells [16].

The presence of CD138-positive plasma cells/10 high power fields (HPFs) associated with pathogenic organisms was considered the gold standard for diagnosing CE [5]. 


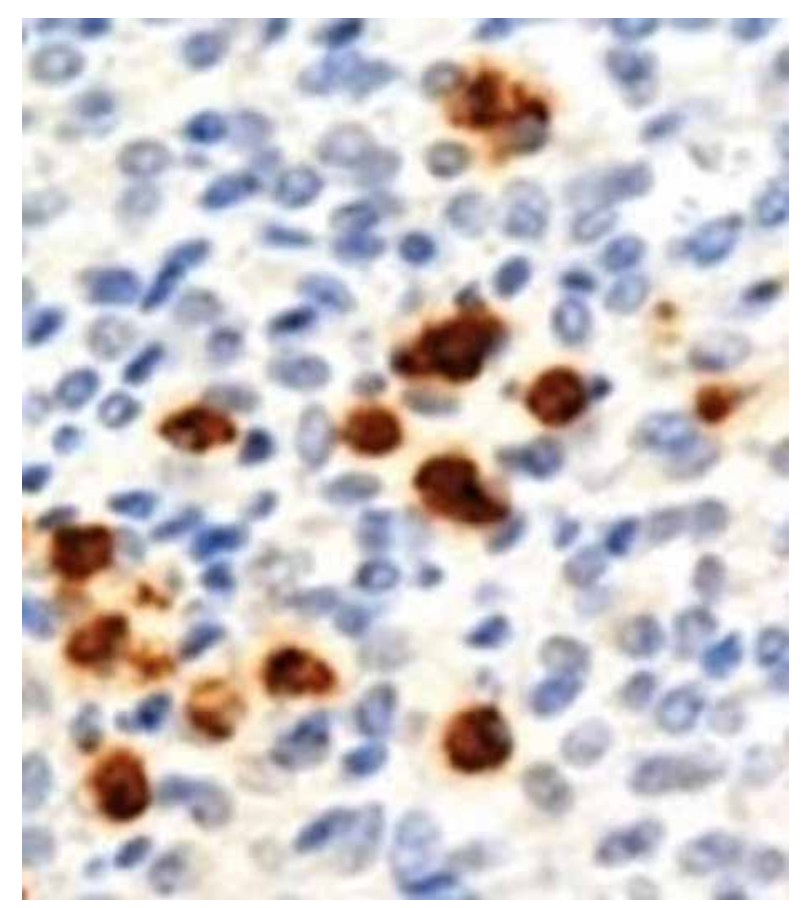

Fig. 3. Immunohistochemistry staining shows grade II chronic endometritis > 5 plasma cells/HPF $(40 \times)$

Table 1. Demographic data of participants, number of previous miscarriages, and type of recurrent miscarriage

\begin{tabular}{lc}
\hline \multicolumn{1}{c}{ Variables } & $\begin{array}{c}\text { Studied participants } \\
\text { (number 110) }\end{array}$ \\
\hline Maternal age (years) & $32.8 \pm 4.2$ \\
\hline BMI $\left(\mathrm{kg} / \mathrm{m}^{2}\right)$ & $26.3 \pm 6.7$ \\
\hline Number of previous miscarriages & $68 / 110(61.8 \%)$ \\
\hline 3 miscarriages & $36 / 110(32.7 \%)$ \\
\hline 4 miscarriages & $6 / 110(5.5 \%)$ \\
\hline$>4$ miscarriages & $71 / 110(64.5 \%)$ \\
\hline $\begin{array}{l}\text { Type of RM } \\
\text { Early RM (> 6 weeks and before } \\
\text { ultrasound diagnosis) }\end{array}$ & $39 / 110(35.5 \%)$ \\
\hline $\begin{array}{l}\text { Clinical RM (after ultrasound } \\
\text { diagnosis) }\end{array}$
\end{tabular}

Data presented as mean \pm SD (standard deviation) and number and percentage (\%)

$\mathrm{BMI}$ - body mass index, $\mathrm{RM}$ - recurrent miscarriage

In addition, the CE was graded according to plasma cell number into: 1-5 plasma cells (grade-I CE), 5-20 plasma cells (grade-II CE), and > 20 plasma cells (grade-III CE) (Fig. 3) [8].

The endometrial biopsies taken for cultures were cultured for the common infectious pathogens, and for Neisseria gonorrhea, Chlamydia trachomatis, Mycoplasma species, Ureaplasma urealyticum, and yeast. Studied women received antibiotics based on the causative pathogens, and antibiogram results.

Collected data were statistically analyzed to assess the relation between CE and RM (primary outcome) and the accuracy of CE-related hysteroscopic signs (micropolyps, stromal edema, and hyperemia) in diagnosing CE (secondary outcome).

\section{Sample size}

The required sample size was calculated using G Power software version 3.1.9.4 for sample size calculation, setting $\alpha$-error probability at 0.05 , power $(1 \beta$-error probability) at $0.95 \%$, and effective sample size (w) at 0.5 . An effective sample size $\geq 110$ women was needed to produce a statistically acceptable figure.

\section{Statistical analysis}

Collected data were statistically analyzed using SPSS computer software version 20 IBM Corp., Armonk (Chicago, IL, USA). Numerical variables were presented as mean and standard deviation ( \pm SD), while categorical variables were presented as frequencies $(n)$ and percentage (\%). The chi-square $\left(\chi^{2}\right)$ test was used to compare qualitative variables.

Sensitivity: proportional detection of individuals with the disease of interest in the population. Specificity: proportional detection of individuals without the disease of interest in the population. Positive predictive value (PPV): proportion of all individuals with positive tests who have the disease. Negative predictive value (NPV): proportion of all individuals with negative tests who are non-diseased. The $p$-value $<0.05$ was considered significant.

\section{Results}

One hundred and ten (110) women aged 20-40 years, with unexplained RM, were included in this study to assess the relation between CE and RM and the accuracy of CE-related hysteroscopic signs in diagnosing CE.

The mean age of the studied women was $32.8 \pm 4.2$ years, mean body mass index (BMI) was $26.3 \pm 6.7 \mathrm{~kg} / \mathrm{m}^{2}$, $61.8 \%(68 / 110)$ of the studied women had 3 previous miscarriages, $32.7 \%(36 / 110)$ had 4 previous miscarriages, and $5.5 \%$ had $>4$ previous miscarriages. About $64.5 \%(71 / 110)$ of the studied women had early RM (> 6 weeks), and 35.5\% (39/110) had clinical RM (after ultrasound diagnosis) (Table 1).

The prevalence of CE in women with RM was $31.8 \%$ (35/110) using CE-related hysteroscopic signs, while it was $38.2 \%(42 / 110)$ using IHC staining and endometrial cultures ( $p=0.4$; insignificant difference).

The prevalence of CE in women with RM was $31.8 \%$ (35/110) using CE-related hysteroscopic signs and included hyperemia (focal or diffuse) in $65.7 \%$ (23/35), stromal edema in 20\% (7/35), and micropolyps in $14.3 \%$ $(5 / 35)$. 
Table 2. Hysteroscopic, immunohistochemistry, culture results, and accuracy of chronic endometritis-related hysteroscopic signs in diagnosing chronic endometritis

\begin{tabular}{|c|c|}
\hline Variables & $\begin{array}{l}\text { Positive CE-related hysteroscopic signs (number } 35 \text { ) } \\
\text { Positive CE by IHC and endometrial culture (number } 42 \text { ) }\end{array}$ \\
\hline CE-related hysteroscopic signs & $35 / 110(31.8)$ \\
\hline Hyperemia (focal or diffuse) & $23 / 35(65.7)$ \\
\hline Stromal edema & $7 / 35(20)$ \\
\hline Micropolyps (< $2 \mathrm{~mm})$ & $5 / 35(14.3)$ \\
\hline CE diagnosed by IHC & $42 / 110(38.2)$ \\
\hline Garde I & $11 / 42(26.2)$ \\
\hline Grade II & $23 / 42(54.8)$ \\
\hline Grade III & $8 / 42(19)$ \\
\hline Endometrial culture results & $42 / 110(38.2)$ \\
\hline Mycoplasmas hominis/Ureaplasma & $11 / 42(26.2)$ \\
\hline Enterococcus faecalis & $9 / 42(21.4)$ \\
\hline Chlamydia trachomatis & $8 / 42(19)$ \\
\hline Escherichia coli & $7 / 42(16.7)$ \\
\hline Streptococcus viridans & $3 / 42(7.1)$ \\
\hline Streptococcus bovis & $2 / 42(4.8)$ \\
\hline Hemolytic staphylococcus & $1 / 42(2.4)$ \\
\hline Candida albicans & $1 / 42(2.4)$ \\
\hline \multicolumn{2}{|c|}{ Accuracy of CE-related hysteroscopic signs in diagnosing CE } \\
\hline Sensitivity $(T P \div T P+F N) \times 100$ & $(25 \div 25+14) \times 100=64.1$ \\
\hline Specificity $(T N \div T N+F P) \times 100$ & $(61 \div 61+10) \times 100=85.9$ \\
\hline$P P V(T P \div T P+F P) \times 100$ & $(25 \div 25+10) \times 100=71.4$ \\
\hline$N P V(T N \div T N+F N) \times 100$ & $(61 \div 61+14) \times 100=81.3$ \\
\hline Accuracy $(T P+T N \div T P+T N+F P+F N) \times 100$ & $(25+61 \div 25+61+10+14) \times 100=78.2$ \\
\hline
\end{tabular}

Data presented as number and percentage (\%).

$\mathrm{CE}$ - chronic endometritis, FN - false negative, FP - false positive, IHC - immunohistochemistry, NPV - negative predictive value, PPV - positive predictive value, $\mathrm{TN}$ - true negative, TP - true positive

The prevalence of CE in women with RM was $38.2 \%$ (42/110) using IHC staining and endometrial cultures; grade-I CE in 26.2\% (11/42), grade-II CE in $54.8 \%$ (23/42), and grade-III CE in 19\% (8/42).

The endometrial cultures showed: Mycoplasmas hominis/Ureaplasma urealyticum in $26.2 \%$ (11/42) of participants, Enterococcus faecalis in 21.4\% (9/42), Chlamydia trachomatis in 19\% (8/42), Escherichia coli in $16.7 \%$ (7/42), Streptococcus viridans in $7.1 \%$ (3/42), Streptococcus bovis in $4.8 \%$ (2/42), Hemolytic staphylococcus in $2.4 \%(1 / 42)$, and Candida albicans in $2.4 \%$ (1/42) (Table 2).

Compared with IHC staining and endometrial cultures (gold standard), CE-related hysteroscopic signs were true positive (TP) in 25 of the studied RM women, and false positive (FP) in 10 cases, while CE-related hysteroscopic signs were true negative (TN) in 61 of the studied RM women, and false negative (FN) in 14 cases. CE-related hysteroscopic signs had $64.1 \%$ sensitivity, 85.9\% specificity, $71.4 \%$ PPV, $81.3 \%$ NPV, and 78.2\% overall accuracy in diagnosing CE (Table 2, Fig. 4).
Compared with IHC staining, and endometrial cultures, CE-related hysteroscopic signs had significantly lower sensitivity $(p=0.03)$, statistically similar specificity, PPV, NPV, and overall accuracy in diagnosing CE $(p=0.4,0.1,0.3,0.2$; respectively).

\section{Discussion}

Growing interest has focused on the relation between CE or asymptomatic inflammation of the endometrium and RM [4].

Identification of plasma cells in the endometrial stroma associated with pathogenic organisms is the gold standard for diagnosing CE [3, 6-8].

Previous studies concluded that the hysteroscopic diagnosis of CE should be based on identification of micropolyps ( $<2 \mathrm{~mm}$ ), stromal edema, hyperemia (focal or diffuse), or hemorrhagic spots during uterine cavity and endometrium evaluation [9-12].

Recently, Drizi et al. concluded that the histological diagnosis of CE should be based on identification 


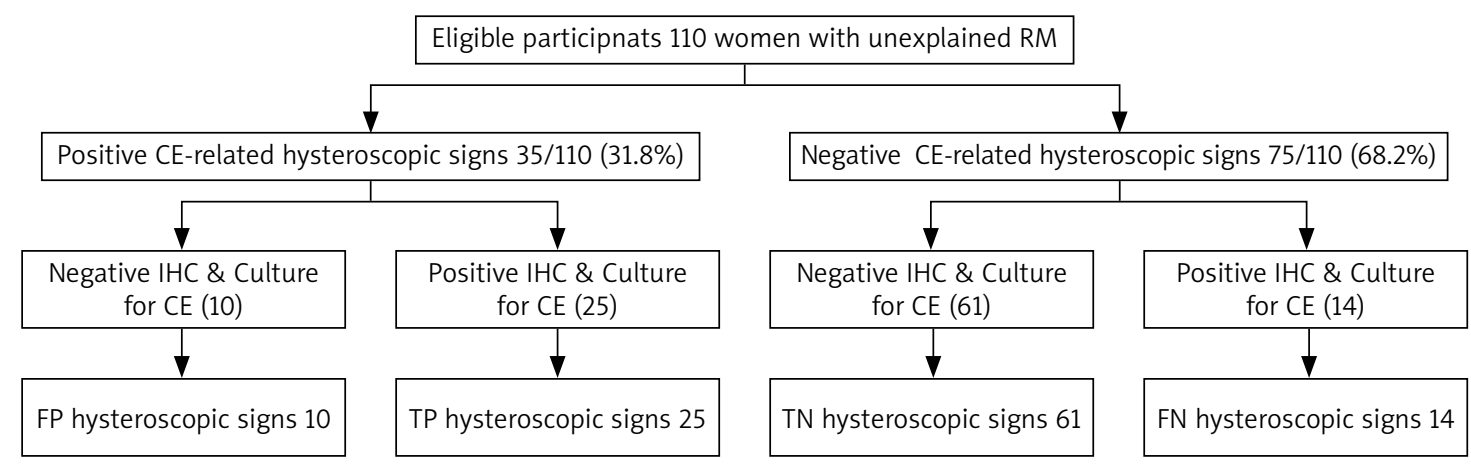

Fig. 4. Study flow-chart, and chronic endometritis related hysteroscopic signs compared to immunohistochemistry and culture results

$\mathrm{CE}$ - chronic endometritis, FN - false negative, FP - false positive, IHC - immunohistochemical staining, RM - recurrent miscarriage, TN - true negative, TP - true positive

of plasma cells, which are normally absent or low in the endometrium, and the hysteroscopic diagnosis of CE should be based on identification of micropolyps, stromal edema, focal hyperemia, or hemorrhagic spots during uterine cavity and endometrium evaluation [12].

Therefore, this comparative double-blinded study was designed to assess the relation between CE and RM and the accuracy of CE-related hysteroscopic signs in diagnosing $C E$.

One hundred and ten (110) women with unexplained RM were identified from the hospital records and included in this study. About $61.8 \%$ (68/110) of the studied women had 3 previous miscarriages, $32.7 \%$ (36/110) had 4 previous miscarriages, and $5.5 \%$ had $>4$ previous miscarriages.

The RM was early RM in $64.5 \%(71 / 110)$ of the studied women, and it was clinical RM in $35.5 \%(39 / 110)$ of them.

The prevalence of CE in women with RM was 31.8\% (35/110) using CE-related hysteroscopic signs, while the prevalence of CE in women with RM was 38.2\% (42/110) using IHC staining and endometrial cultures $(p=0.4)$.

Similarly, Zargar et al. found that the prevalence of CE in RMs was $36.8 \%$ and $31.6 \%$ based on hysteroscopy and IHC staining, respectively [17].

Kimura et al. reported a $9.3-67.6 \%$ prevalence of CE in RM [5]. Bouet et al. reported a $27 \%$ prevalence of CE in RM [6], and Kaku et al. found plasma cell clusters in more than $50 \%$ of women with CE [8].

Kaku et al. concluded that the presence of plasma cell clusters had $53.8 \%$ sensitivity, $100 \%$ specificity, $100 \%$ PPV, and 68.4\% NPV in diagnosing CE [8].

The difference in prevalence of CE between studies was explained by McQueen et al., who found higher prevalence of CE with IHC staining compared to traditional hematoxylin and eosin staining (56\% versus 13\%) [7].

Zargar et al. found that hysteroscopy had $86.36 \%$ sensitivity, $87.3 \%$ specificity, $70.37 \%$ NPV, and $94.82 \%$ PPV in diagnosing CE [17].

This study found that CE-related hysteroscopic signs had $64.1 \%$ sensitivity, $85.9 \%$ specificity, $71.4 \%$ PPV,
81.3\% NPV, and $78.2 \%$ overall accuracy in diagnosing CE compared with IHC staining and endometrial cultures (gold standard).

Compared with IHC staining and endometrial cultures, CE-related hysteroscopic signs had significantly lower sensitivity $(p=0.03)$, statistically similar specificity, PPV, NPV, and overall accuracy in diagnosing CE ( $p=0.4,0.1,0.3,0.2$; respectively).

In addition, Liu et al. found that the hysteroscopic scoring system based on endometrial hyperemia, dilated endometrial vessels, endometrial micropolyps, and polypoidal hyperplasia had $62.8 \%$ sensitivity, $91.7 \%$ specificity, $88.8 \%$ PPV, and $70.1 \%$ NPV in diagnosing CE [18].

Moreover, Song et al. found that the presence of $\geq$ one of the CE-related hysteroscopic signs had $59.3 \%$ sensitivity, $69.7 \%$ specificity, $42.1 \%$ PPV, $82.8 \%$ NPV, and $66.9 \%$ accuracy in diagnosing CE [19].

The results of endometrial culture showed: $\mathrm{Myco-}$ plasmas hominis/Ureaplasma in 26.2\% (11/42) of participants, Enterococcus faecalis in 21.4\% (9/42), Chlamydia trachomatis in 19\% (8/42), Escherichia coli in $16.7 \%$ (7/42), Streptococcus viridans in 7.1\% (3/42), Streptococcus bovis in $4.8 \%$ (2/42), Hemolytic staphylococcus in $2.4 \%$ (1/42), and Candida albicans in 2.4\% (1/42).

Cicinelli et al. also found that common bacteria were the causative pathogens in $77.5 \%$ of CE, Mycoplasma and Ureaplasma in $25.3 \%$ of CE, and Chlamydia trachomatis in $12.7 \%$ of CE [3].

Cicinelli et al., in another study, found that $>70 \%$ of CE cases were caused by common pathogens and Mycoplasma, and not by Chlamydia trachomatis or N. gonorrhoeae [11].

Participants with CE by confirmed IHC staining and endometrial cultures received antibiotics based on the causative pathogens, and antibiogram results to improve the pregnancy outcome. Kimura et al. concluded that antibiotics are an effective therapeutic option for $\mathrm{CE}$, and the pregnancy rate significantly improved when CE was treated with antibiotics [5].

A recent SWOT analysis also recommended investigating and managing CE in women with RPL or repeated 
implantation failure after in vitro fertilization as it could improve outcomes [20].

This study found that the prevalence of CE in women with RM was $31.8 \%$ using CE-related hysteroscopic signs, while it was $38.2 \%$ using $\mathrm{IHC}$ staining and endometrial cultures $(p=0.4)$.

CE-related hysteroscopic signs had $64.1 \%$ sensitivity, $85.9 \%$ specificity, $71.4 \%$ PPV, $81.3 \%$ NPV, and $78.2 \%$ overall accuracy in diagnosing CE compared with $\mathrm{IHC}$ staining and endometrial cultures (gold standard). Compared with IHC staining and endometrial cultures, CE-related hysteroscopic signs had significantly lower sensitivity ( $p=0.03)$, and statistically similar specificity, PPV, NPV, and overall accuracy in diagnosing CE ( $p=0.4$, $0.1,0.3,0.2$; respectively).

Most cases of CE (> 81\%) were caused by Mycoplasma and common pathogens (not caused by Chlamydia trachomatis or $N$. gonorrhoeae).

Women refusing to participate and/or give consent, small sample size, retrospective nature of the study, and absence of controls (without previous miscarriage) and follow-up after treatment were the limitations of this study.

Despite these limitations, this study was the first registered comparative double-blinded study conducted to assess the relation between CE and RM and the accuracy of CE-related hysteroscopic signs in diagnosing $\mathrm{CE}$.

In addition, there are no clear guidelines on whether to include CE in the initial assessment of an infertile couple [20]. Therefore, future randomized trials are needed to determine whether to include CE in the initial assessment of an infertile couple, and the impact of antibiotic therapy in CE on endometrial receptivity/ implantation.

\section{Conclusions}

The prevalence of CE in women with RM was $31.8 \%$ using CE-related hysteroscopic signs, while it was $38.2 \%$ using IHC staining and endometrial cultures. Compared with IHC staining and endometrial cultures, CE-related hysteroscopic signs had significantly lower sensitivity, and statistically similar specificity, PPV, NPV, and overall accuracy in diagnosing CE. Hysteroscopy is a useful complementary tool to endometrial culture and IHC staining in diagnosing CE. Future randomized trials are needed to determine whether to include CE in the initial assessment of an infertile couple and the impact of antibiotic therapy in CE on endometrial receptivity/implantation.

\section{Acknowledgments}

This study was approved by the Obstetrics and Gynecology Department ethical committee and registered as a clinical trial.
The authors are grateful to the women who agreed and gave consent to participate in this study.

\section{Disclosure}

The authors report no conflicts of interest.

\section{References}

1. Jauniaux E, Farquharson RG, Christiansen OB, Exalto N. Evidence-based guidelines for the investigation and medical treatment of recurrent miscarriage. Hum Reprod 2006; 21: 2216-2222.

2. RCOG Green Top Guideline. The investigation and treatment of couples with recurrent first trimester and second-trimester miscarriage. RCOG Green Top Guideline 2011; 17: 1-17.

3. Cicinelli E, Matteo M, Tinelli R, et al. Chronic endometritis due to common bacteria is prevalent in women with recurrent miscarriage as confirmed by improved pregnancy outcome after antibiotic treatment. Reprod Sci 2014; 21: 640-647.

4. Greenwood SM, Moran JJ. Chronic endometritis: morphologic and clinical observations. Obstet Gynecol 1981; 58: 176-184.

5. Kimura F, Takebayashi A, Ishida M, et al. Review: chronic endometritis and its effect on reproduction. J Obstet Gynaecol Res 2019; 45: 951-960.

6. Bouet PE, El Hachem H, Monceau E, Gariépy G, Kadoch IJ, Sylvestre C. Chronic endometritis in women with recurrent pregnancy loss and recurrent implantation failure: prevalence and role of office hysteroscopy and immunohistochemistry in diagnosis. Fertil Steril 2016; 105 : 106-110.

7. McQueen DB, Perfetto CO, Hazard FK, Lathi RB. Pregnancy outcomes in women with chronic endometritis and recurrent pregnancy loss. Fertil Steril 2015; 104: 927-931.

8. Kaku S, Kubo T, Kimura F, et al. Relationship of chronic endometritis with chronic deciduitis in cases of miscarriage. BMC Womens Health 2020; 20: 114.

9. Cicinelli E, Resta L, Nicoletti R, Zappimbulso V, Tartagni M, Saliani N. Endometrial micropolyps at fluid hysteroscopy suggest the existence of chronic endometritis. Hum Reprod 2005; 20: 1386-1389.

10. Cicinelli E1, Resta L, Nicoletti R, et al. Detection of chronic endometritis at fluid hysteroscopy. J Minim Invasive Gynecol 2005; 12: 514-518.

11. Cicinelli E, De Ziegler D, Nicoletti R, et al. Chronic endometritis: correlation among hysteroscopic, histologic, and bacteriologic findings in a prospective trial with 2190 consecutive office hysteroscopies. Fertil Steril 2008; 89: 677-684.

12. Drizi A, Djokovic D, Laganà AS, van Herendael B. Impaired inflammatory state of the endometrium: a multifaceted approach to endometrial inflammation. Current insights and future directions. Prz Menopauz 2020; 19: 90-100.

13. http://www.ANZCTR.org.au/ACTRN12618000561280.aspx.

14. Saravelos SH, Li TC. Unexplained recurrent miscarriage: how can we explain it? Hum Reprod 2012; 27: 1882-1886.

15. Resta L, Palumbo M, Rossi R, Piscitelli D, Grazia Fiore M, Cicinelli E. Histology of micro polyps in chronic endometritis. Histopathol 2012; 60: 670-674. 10.1111/j.1365-2559.2011.04099.x.

16. Kannar V, Lingaiah HK, Sunita V. Evaluation of endometrium for chronic endometritis by using syndecan-1 in abnormal uterine bleeding. J Lab Physicians 2012; 4: 69-73.

17. Zargar M, Ghafourian M, Nikbakht R, Mir Hosseini V, Moradi Choghakabodi P. Evaluating chronic endometritis in women with recurrent implantation failure and recurrent pregnancy loss by hysteroscopy and immunohistochemistry. J Minim Invasive Gynecol 2020; 27: 116-121.

18. Liu $\mathrm{H}$, Song J, Zhang F, et al. A new hysteroscopic scoring system for diagnosing chronic endometritis. J Minim Invasive Gynecol 2020; 27: 1127-1132.

19. Song D, Li TC, Zhang Y, et al. Correlation between hysteroscopy findings and chronic endometritis. Fertil Steril 2019; 111: 772-779.

20. Espinós JJ, Fabregues F, Fontes J, et al. Spanish Infertility SWOT Group (SISG). Impact of chronic endometritis in infertility: a SWOT analysis. Reprod Biomed Online 2021; 42: 939-951. 